\title{
Bonding Strength Analysis of Multi-material and Multi-color Specimens Printed with Multi-extrusion Printer
}

Gani Issayev (0000-0002-2072-1951), Aidos Aitmaganbet (0000-0002-7439- 305X ),

Essam Shehab (0000-0003-3593-6944), Md. Hazrat Ali (0000-0003- 0428-957X)

Department of Mechanical and Aerospace Engineering, SEDS, Nazarbayev University, Nur-Sultan 010000, Kazakhstan, E-mail:md.ali@nu.edu.kz

This paper highlights the findings of multi-material application in 3D printed specimens. The work presents experimental results of multi-material, and multi-color-based 3D printed specimens by a multiextrusion printer developed at the laboratory. It aimed to determine the bonding properties between the layers of various materials indicated with different colors. The samples were printed across multiple infill models and tested in the tensile and compression machine. The specimens were created with $10 \%, 25 \%$, and $\mathbf{1 0 0 \%}$ infill having single and dual colors material. Polylactic Acid (PLA), Acrylonitrile Butadiene Styrene (ABS), and Flex materials were used for printing various specimens with various colors. The layers were printed synchronously. The filaments were changed according to the predetermined algorithm. The experimental results showed that the mechanical properties of single, dual, and triple material specimens differed according to the reinforcement strategy. In addition, the mechanical property of the same material with different colors was identical.

Keywords: 3D Printing, FDM, Dual-colors, Multi-layers, Infill

\section{Introduction}

Nowadays, additive manufacturing (AM) as a type of advanced manufacturing technology is gaining an increased amount of attention. This is due to the AM's favorable characteristics that enable more cost- and resource-efficient small-scale manufacturing. Moreover, the application of AM technologies can offer advantages, including complex-shaped parts, highly customizable parts, product longevity (through repair, remanufacture, and refurbishment), shorter and simpler supply chains [1].

$3 \mathrm{D}$ printers, as a part of AM technologies, are becoming affordable with the technology advancement. One promising AM technology with a comparably cheaper cost is the Fused Deposition Modeling (FDM). This is also referred to as Fused Filament Fabrication (FFF), Modeling Extrusion (ME), and Fused Layer Manufacturing (FLM). In FDM, the melted material (filament) is extruded through a nozzle and selectively dispensed in a heated support plate $[2,3]$. FDM manufactured parts include tensile test, visual aids, educational models, assembly models, and even fully functional parts [4-6]. Nowadays, the main FDM technology advancement comprises different directions like optimal designing of the parts [7, 8], enhancing mechanical properties of the final product [9], and multi-material printing [10-13]. Particularly, the multi-material 3D printing approach has high poten- tial application opportunities by adding more complexity and functionality, for example, topological optimization with multi-materials usage [11], biomedical applications [14, 15], automotive industries [10], Electronics [16], etc.

Earlier appearances of the multi-material 3D printing can be found in various instances such as:

1) FDM fabrication of actuators and sensors from multiple ceramic materials [17],

2) Manufacturing of the medical scaffolds by a multi-nozzle biopolymer deposition systém $[14,15]$,

3) Fabrication of fully functional electronics with the solid freeform fabrication (SFF) method [16],

4) Building multi-material $3 \mathrm{D}$ parts and electronics with stereolithography (SL) $[18,19]$.

The first professional-grade material extrusion system for discrete multi-material fabrication was designed by Espalin et al. [10]. Here they constructed a multi-material FDM printer by combining two legacy FDM machines. Hence, the newly designed machine had a single manufacturing system consisting of two extrusion nozzles and can deposit two different materials simultaneously. The deposited layers from different nozzles vary in materials and layer thicknesses, and road width.

Baca and Ahmad [13] compared the mechanical properties of the FDM printed parts manufactured by multi-material single nozzle and multi-material multiple nozzles. Tensile testing specimens with rectilinear 
infill patterns and different material layers were printed from both FDM nozzle configurations. It was concluded that the multi-nozzle FDM can be beneficial in terms of building time, whereas the single-nozzle FDM can be used to fabricate high-quality parts. In addition, the results of mechanical property tests revealed only a minor difference.

Despite all the advantages listed above, the multimaterial FDM technique possesses a major drawback. This limitation is caused by the discontinuity of the FDM process and results in a poor mechanical strength of the fabricated part [20]. Subsequently, this may even severely affect multi-material FDM parts where the detachment of the layers can happen [21].

A multi-nozzle extrusion system for a multi-material FDM printer was first pioneered by our group [12, $22]$. We proposed the novel design and working principle of the extruder, which can deposit multi-color as well as multi-material filaments simultaneously. The designed extruder configuration allowed the printing process to continue without stoppings for the filament changes. This way, the printing time was significantly reduced. Furthermore, driving parts and hot-end parts were separated by using the Bowden-type extruder.

In order to further analyze the viability of multinozzle, multi-material, multi-color 3D printing, we have designed and built a new FDM printer based on our previous works $[12,22]$. Therefore, the key objectives of this paper are to describe and introduce the new FDM printer's working principle and analyze the quality of the printed parts by tensile and compression tests. In this context, the newly designed and built FDM printer is briefly discussed. Then, mechanical properties such as tensile and compression test results are discussed for the single-colored and materials as well as multi-colored and materials specimens with different infills. Finally, the bonding characteristics are discussed for teh above specimens.

\section{Experimental Apparatus and Methodol- ogy}

\subsection{Newly developed multi-nozzle FDM printer}

During this research, the first of its kind FDM printer with five nozzles was developed and created. This extrusion system consists of a hot section and a cold section. The hot section contains five nozzles for five different colors/materials, fixed with thermal barriers to the holding plate in a circular pattern. Each nozzle has a heater and a heat sensor to provide a uniform temperature for material melting. The main feature of this system is that a separate material filament is used for each nozzle. Because of this, there is no need to change the filament manually since all five filaments are attached simultaneously. This helps to reduce printing time as well as the frequency of printer maintenance and cleaning. The main purpose of the cold section of this extrusion system is to feed the material and switch between filaments. The material is fed using a gear that is connected to a stepper motor. A pushing mechanism consisting of spring provides sufficient contact between the filament and the gear. There are also two additional stepper motors. In combination with proximity sensors, the first motor is used to switch between the nozzles, and the second lighter stepper motor is used to move the gear simultaneously. Since this system uses only three motors, the entire structure is lightweight. This provides a faster and an easier movement in all three axes, which is another advantage of this printer.

\subsection{Specimen fabrication}

The tensile test specimens were manufactured according to the ASTM D638 Type V. The dimensions are shown in Fig. 1 (all dimensions are in $\mathrm{mm}$ ). Whereas the compression test specimens were manufactured as a cylinder with a diameter of $20 \mathrm{~mm}$ and a height of $20 \mathrm{~mm}$. In this work, the process parameters such as materials, color (from different nozzles), and infill rate were varied to investigate their effect on the tensile as well as compression strength characteristics. This method allows studying the bonding properties among different colors and materials from various nozzles with unidentical infill rates. Three key materials are used; namely, PLA, ABS, and Flex, to create the same specimens with identical structure and dimensions.
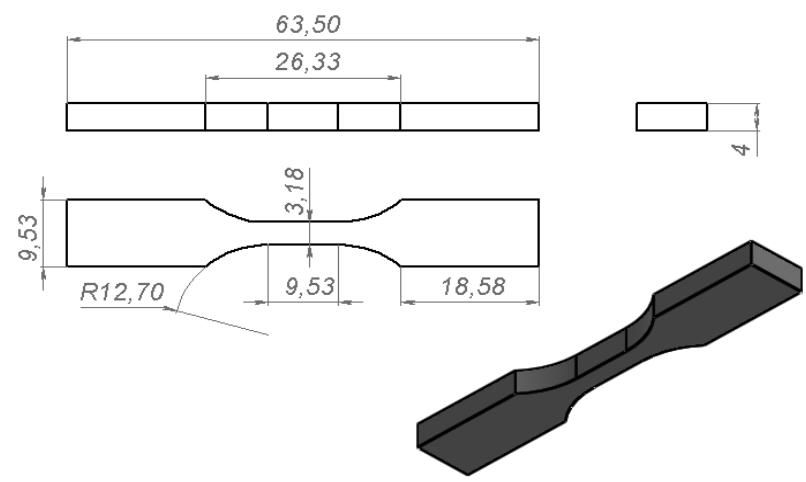

Fig. 1 Tensile specimen dimensions according to ASTM D638-V. All dimensions are in $\mathrm{mm}$.

Figure 2 depicts $10 \%, 25 \%$, and $100 \%$ infill rates of the single-colored and two-colored tensile specimens. On the other hand, the compression test cylinders are illustrated in Fig. 3. Similar to tensile specimens, cylinders were manufactured with three different infill rates and two different colors but with the additional multi-layered type.

The parts were modeled in SolidWorks Software and then saved in STL format. Then these files were transferred to the RepetierHost program, which slices the CAD models. Prior to slicing, various print parameters such as nozzle temperature, bed temperature, and print speed were adjusted in RepetierHost. These 
parameters are given in Table 1. After setting these parameters, they were used to slice all the models used in this experiment. The variable parameters for each part were only the number of materials (colors) and the percentage of infill, which was indicated above. The result of the slicing process is a G-Code file that has also been further adapted to the unique type of printer. After preparing the G-Code files, they were used by a Python file with a set of commands for printing. All the parts were fabricated with the same process parameters as given in Table 1. Brown and green PLA filaments were used as a material.

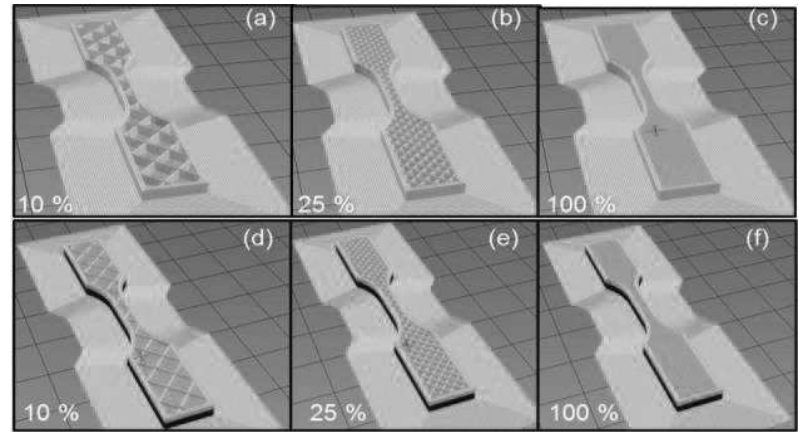

Fig. 2 Tensile specimens with different infill rate $10 \%$, $25 \%, 100 \%$ ) and different colors ( $a, b, c$-single-colored and $d, e, f$ - two-colored specimens)

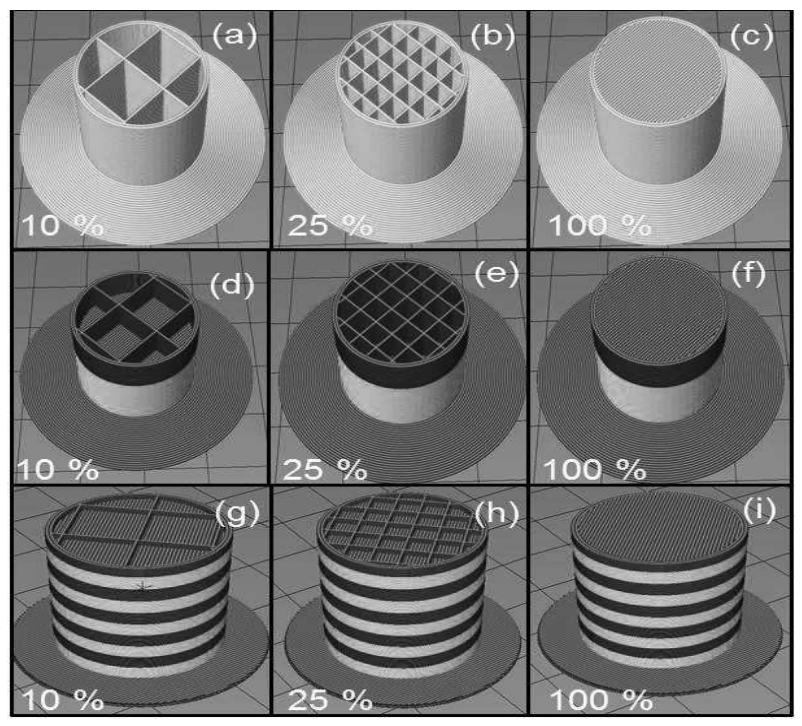

Fig. 3 Cylinders for compression test with different infill rates and different colors (a,b,c-single-colored; d,e,f-two-colored, two-layered; g,h,I - two-colored, multi-layered cylinders).

Tab. 1 FDM printer process parameters

\begin{tabular}{cc}
\hline Parameters & Value \\
\hline Temperature of & $210{ }^{\circ} \mathrm{C}$ \\
Nozzle $\# 1$ & \\
Temperature of & $240{ }^{\circ} \mathrm{C}$ \\
Nozzle $\# 2$ & $60{ }^{\circ} \mathrm{C}$ \\
Bed Temperature & $22 \mathrm{~mm} / \mathrm{s}$ \\
Deposition & 1 \\
Speed & PLA, ABS, and Flex \\
No. of perimeters & Material
\end{tabular}

\subsection{Determination of mechanical properties}

Tensile and compression properties were evaluated by conducting tests with the Tinius Olsen H25KS universal testing machine. The machine can reach a maximum of $25 \mathrm{kN}$ load. The tensile specimen is gripped and tightened by the bottom and upper jigs (Fig. 4a), whereas the compression tests were performed using the compression set-up (Fig.4b). Both tensile and compression tests were done with a speed of 5 $\mathrm{mm} / \mathrm{min}$. Finally, the plots of the load change as a function of displacement were recorded (please refer to Fig. 6).

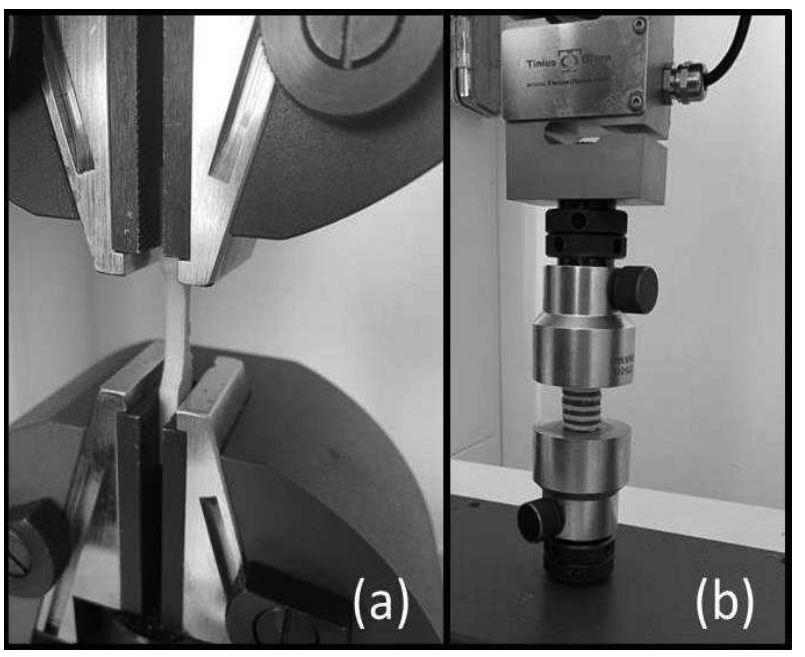

Fig. 4 Application of the Tinius Olsen testing machine for (a) tensile tests and (b) compression tests.

\section{Results and discussion}

\subsection{Case 1: Single material-dual color}

A number of tensile specimens and cylinders were printed by multi-nozzle FDM to evaluate the mechanical properties of 3D printed parts. Broken specimens from tensile and compression tests are represented in Fig. 5.

The following plots ( $6 \mathrm{a}-\mathrm{d})$ represent experimental data of tensile and compression tests. Generally, the tensile and compression curves exhibited similar trends throughout all the tested conditions. However, there were differences in breaking loads and elongation magnitudes. Some of the differences can be expected initially. For instance, as predicted, both colored and single-colored specimens with $100 \%$ infill rates showed similar loads (Fig. 6c) and tensile strength (Fig. 7a). In comparison, two-colored specimens showed higher tensile strength than single-colored ones for 10\% and 25\% infill rates (Fig. 7a). This stems from the fact that two colored specimens were fabricated with thicker outer shells for later infill rates (as shown in Fig. 8). Moreover, from Fig. 7a, the tensile strength ranges from $\approx 21 \mathrm{MPa}$ to $\approx 27 \mathrm{MPa}$ for $100 \%$ infill rates. This perfectly matches the literature tensile strength data of PLA [23]. 


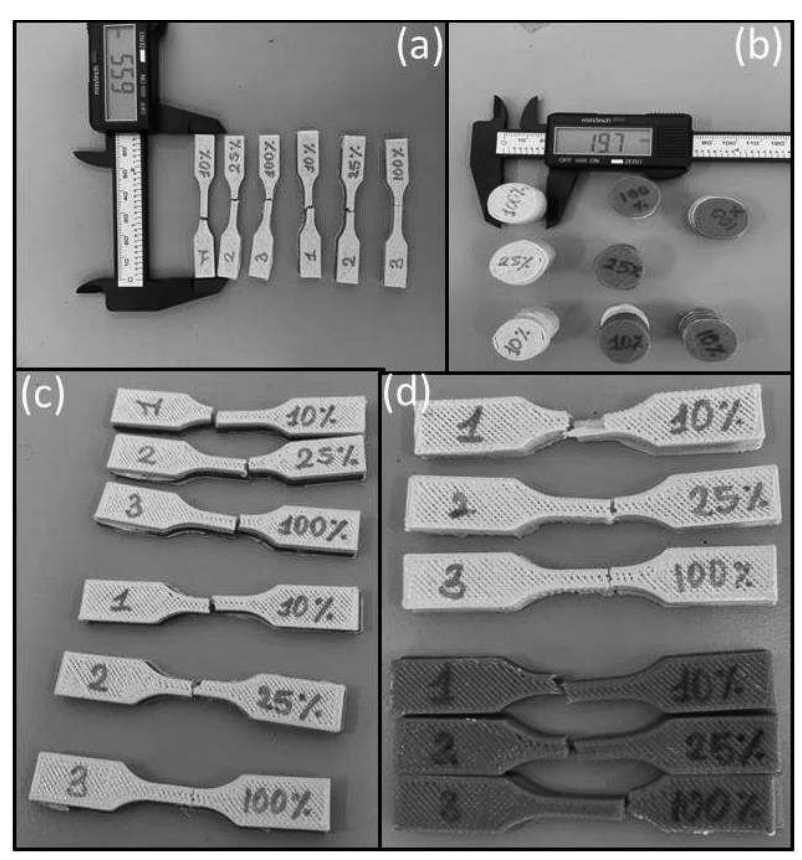

Fig. 5 Failure of tensile and compression tested samples. $a, b$ - dual-colored tensile test specimens, $d$ - single-colored specimens, and $b$-compression test cylinders

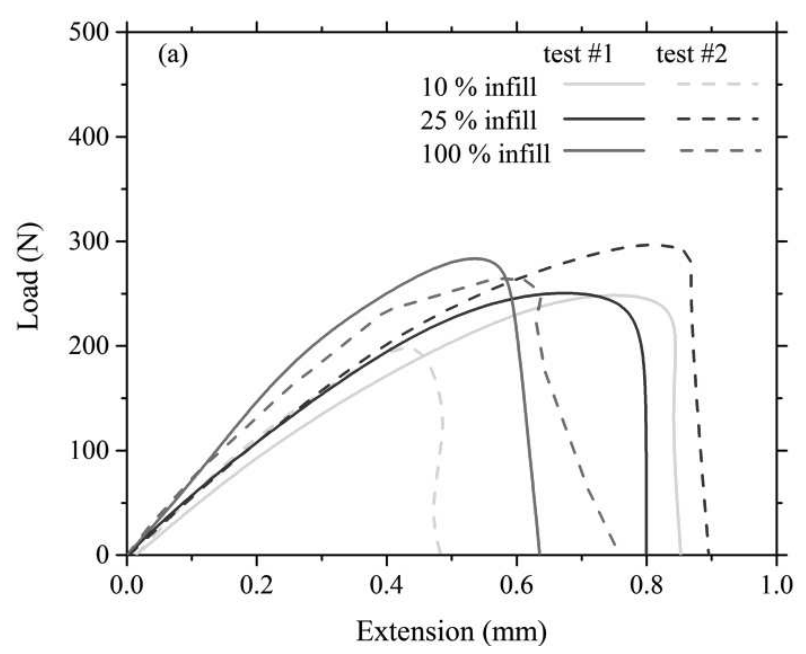

(a)

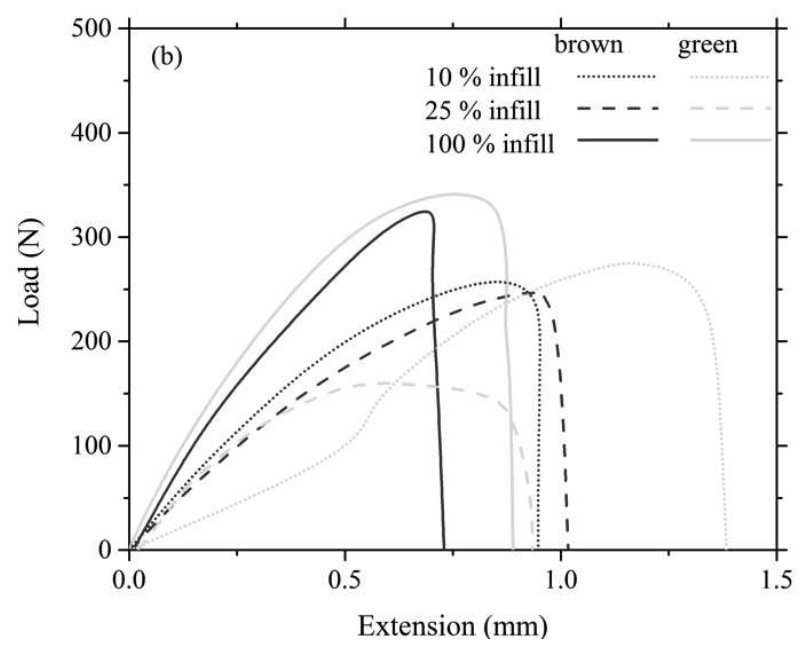

(b)

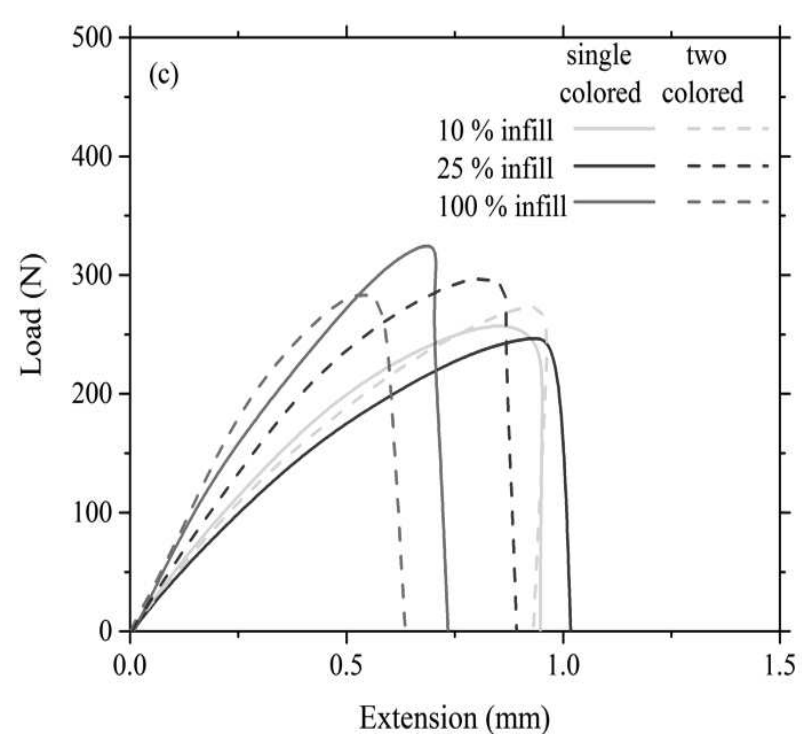

(c)

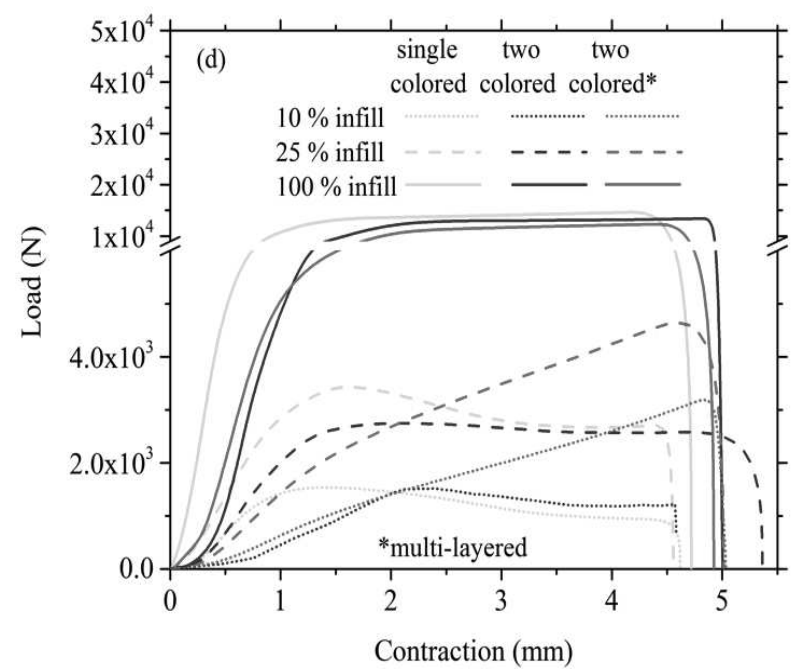

(d)

Fig. 6 Tensile and compression test results. $a-$ two-colored specimens tensile test results (repeatability check), $b$ - tensile test results comparison of different colored specimens (single-colored specimens), $c-$ two colored and single-colored specimen re-

sults comparisons, $d$-compression test results cylinders

The compressive test results indicated that the $100 \%$ infill rates possess the highest compressive strength, followed by $25 \%$, and the least strength has $10 \%$ infill rates (Fig-s $6 \mathrm{~d}$ and $7 \mathrm{~b}$ ). One thing to note is an almost linear increase of the two colored and multilayered compression curves (red dotted and red dashed lines in Fig. 6d). This could be the effect of the bonding of different colors from different nozzles.

Overall, the multi-colored specimens do not demonstrate any mechanical property degradation from the tensile and compressive test results. Consequently, the bonding of materials extruded from different nozzles with different colors was as effective as a single nozzle and single color FDM printing. 

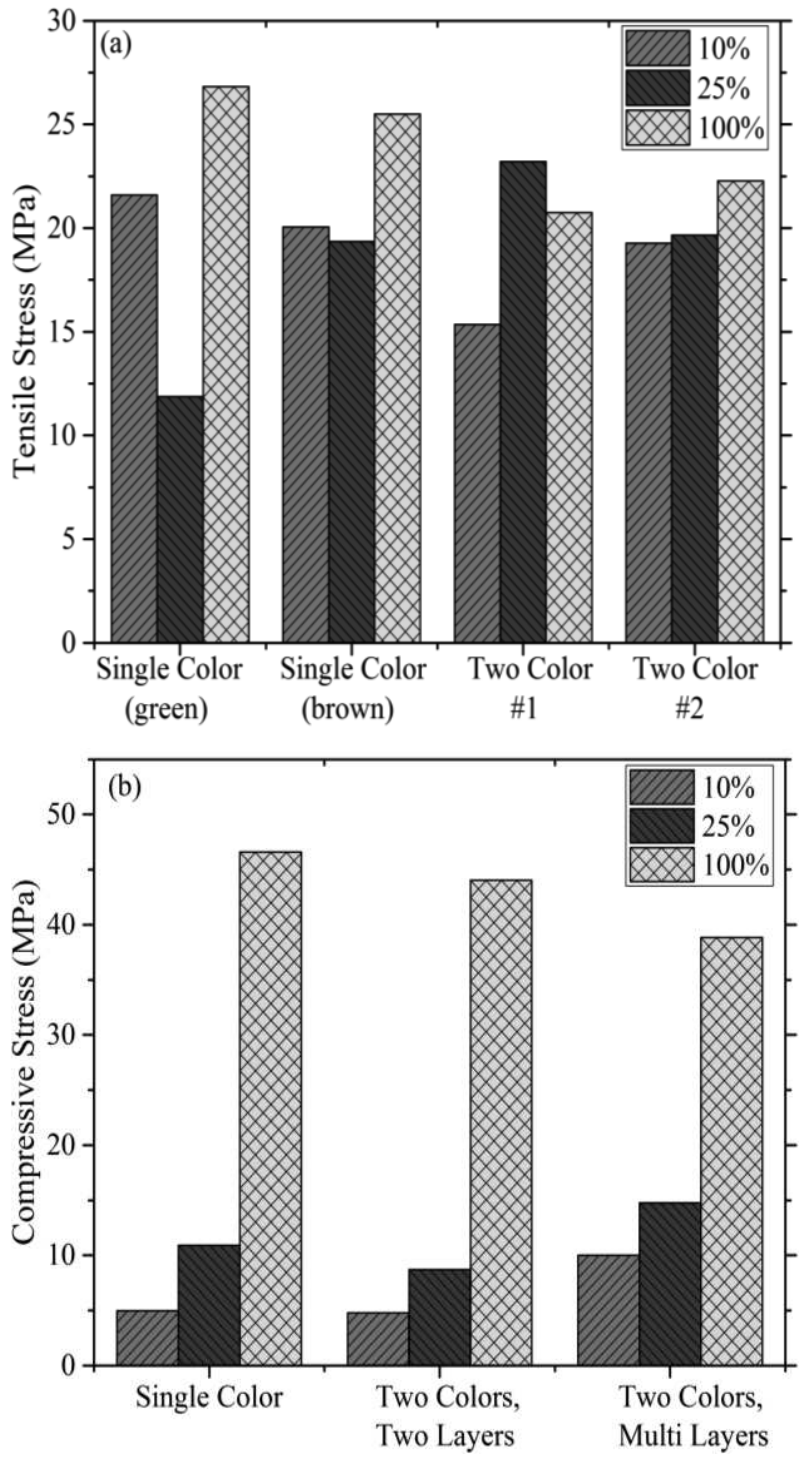

Fig. 7 Calculated tensile (a) and compressive stresses (b)

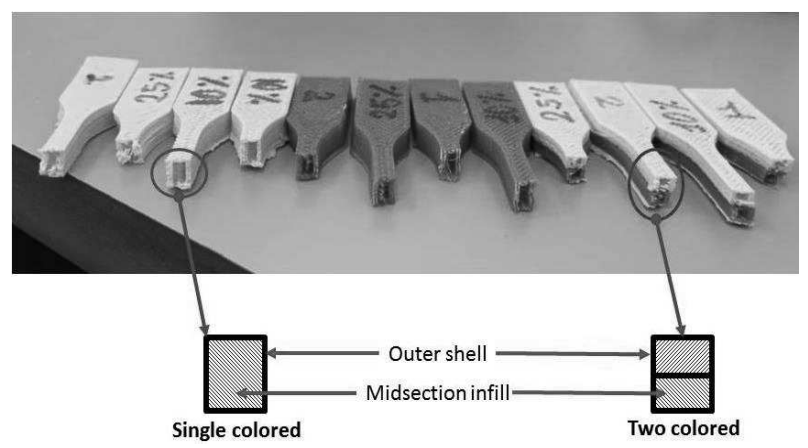

Fig. 8 Tensile specimens section views. Two colored specimens have thicker outer shells than single colored ones

\subsection{Case 2: Multi-material and multi-color}

The muti-material-based printed specimens are shown in Fig. 9. The experimental data were recorded for a distance of $5 \mathrm{~mm}$ from the original specimens' dimensions, either in elongation or compression (Fig. 10), to examine the behaviors in the identical length. Figures 11 (a) - (c) show that the higher infill specimens resulted in better tensile or compression strength than those with less infill rate. Also, the bonding strength was higher in PLA-Flex specimens than the PLA-ABS. The flex material is soft. PLA is softer than ABS. Thus, PLA-Flex has better bonding properties than PLA-ABS. ABS is brittle, and it needs a higher melting temperature than the PLA. Due to the mechanical properties of individual filament, the multi-material specimens' property varies. The breaking point is always found in the bonding areas between different materials.

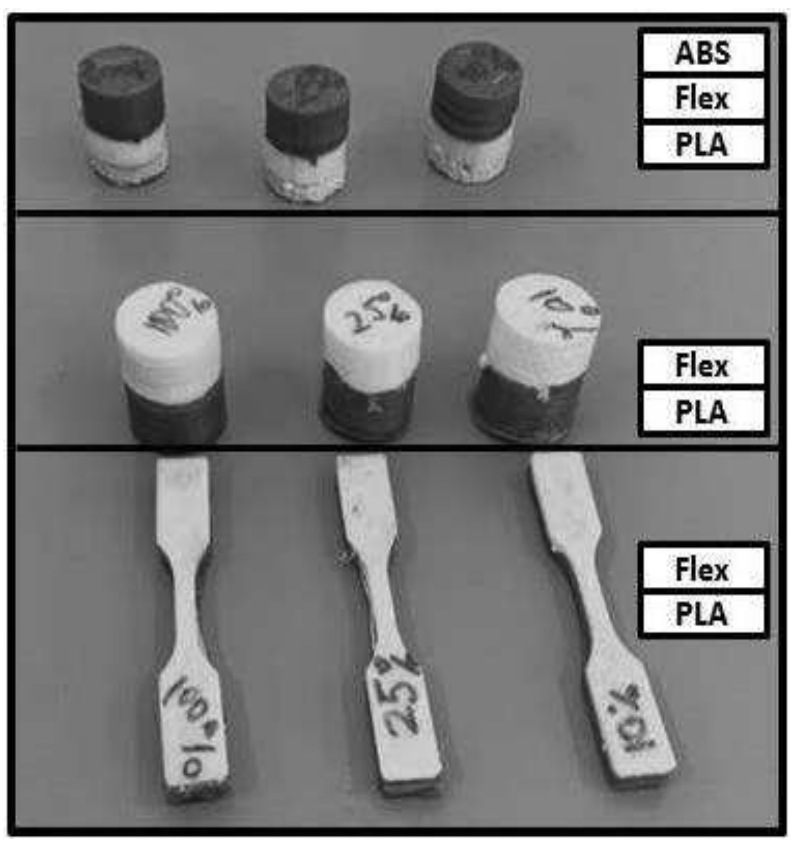

Fig. 9 Multi-material printed cylinders and tensile specimens. Materials used: ABS (blue colored), Flex (yellow-colored), PLA (brown and cyan colored)

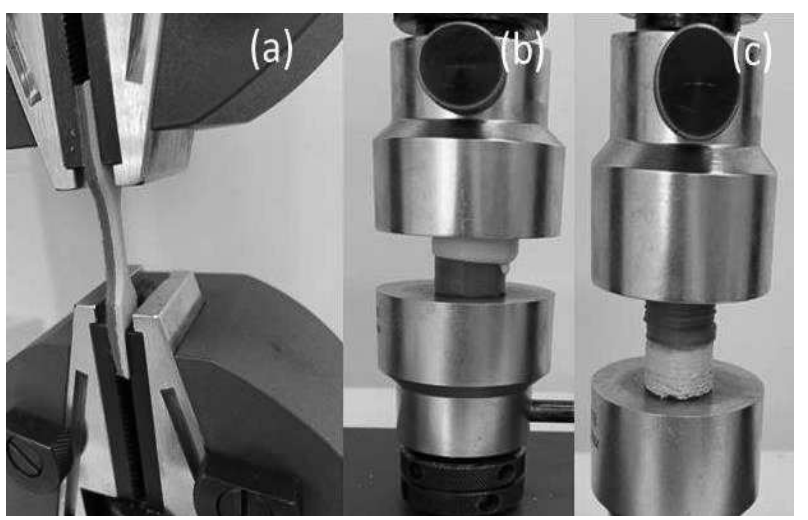

Fig. 10 Application of the Tinius Olsen testing machine for (a) tensile tests of the multi-material specimen (elongation) and (b)-(c) compression tests of multi-material cylinders 

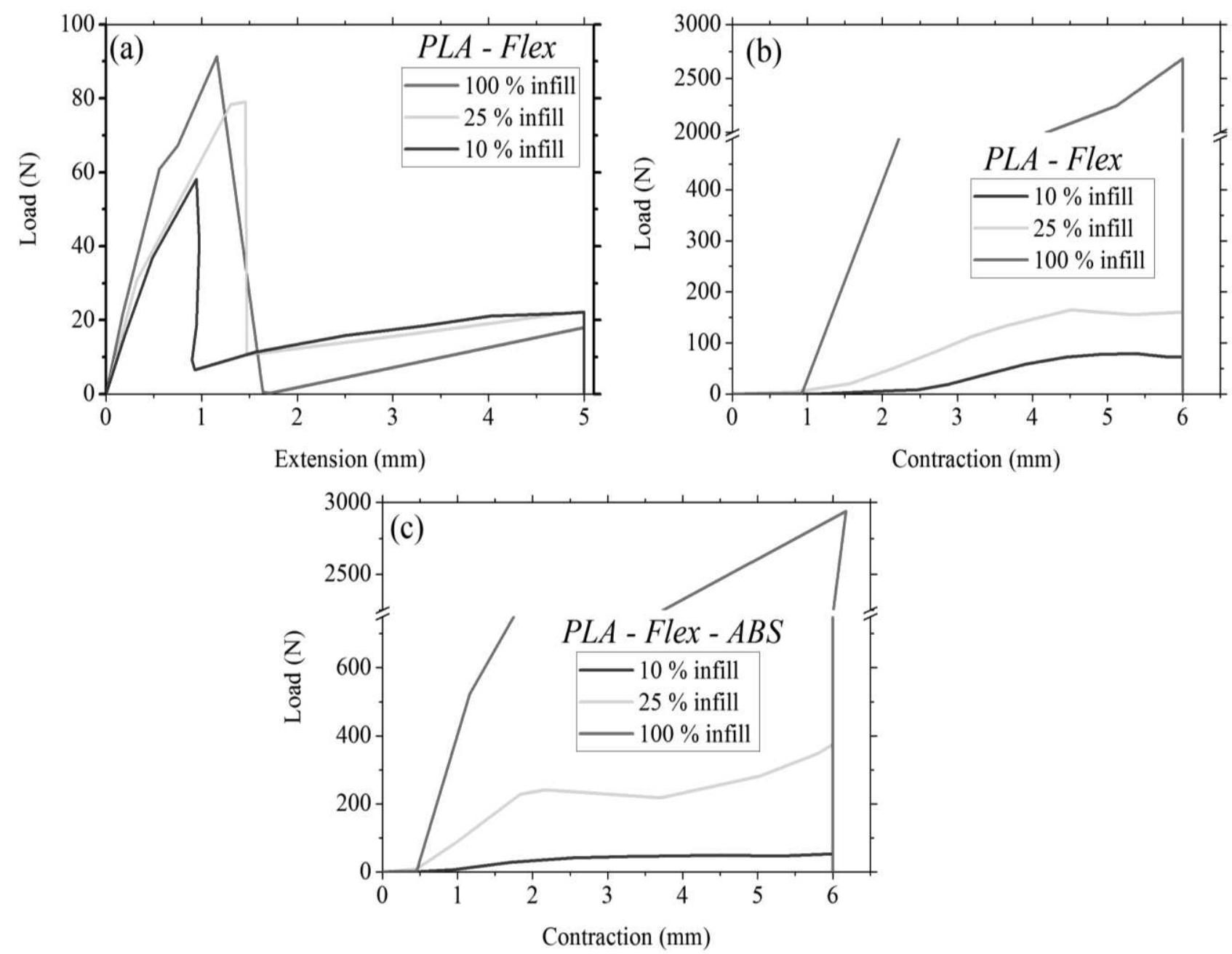

Fig. 11 Experimental results of multi-material specimens: (a) tensile test results of dual material (PLA-Flex) specimens; (b) compression test results of dual material (PLA-Flex) specimens; (c) compression test results of tri material (PLA-Flex-ABS) specimens.

\section{Conclusions}

Specimens for tensile and compression tests were fabricated by a newly designed multi-nozzle and multimaterial FDM 3D printer. Bonding effects of different colors and materials-based filaments were extruded to study the mechanical properties of the printed specimens. The tensile and compression test results showed that the mechanical properties remained similar for single and two colored specimens printed with the same materials. The mechanical properties such as tensile strength and bonding strength were reduced for the specimens printer with PLA-ABS, PLA-Flex, and PLA-Flex-ABS. Less strong bonding properties were observed between the layers of different materials. It was also observed that the bonding strength in PLA-Flex was higher than the PLA-ABS. It could be due to the mechanical properties of those materials. In the next stage, the mechanical properties of multi-material specimens for bigger objects will be investigated. This will further help to analyze multi-material applications in 3D printing technology.

\section{Acknowledgement}

This work was supported by the Faculty Development Competitive Research Grants, Ref. No. 021220FD1551, Nazarbayev University.

\section{References}

[1] S. FORD, M. DESPEISSE (2016). Additive manufacturing and sustainability: an exploratory study of the advantages and challenges, $J$. Clean. Prod. 137 (2016) 1573-1587.

[2] J. STRNAD, M. REZNICEK, K. JELINKOVA, V. JANOSTIK, M. OVSIK (2019). Possibilities of Creating a Mechanism on FDM 3D Printer, Manuf. Technol. J. 19(3) (2019) 508-512.

[3] S. VYAVAHARE, S. TERAIYA, D. PANGHAL, S. KUMAR (2020). Fused deposition modelling: a review, Rapid Prototyp. J. 26(1) (2020) 176-201. 
[4] S. CRUMP (1992). Rapid prototyping using FDM, Mod. Cast. 82(4) (1992) 36-38.

[5] J.Y. WONG, A.C. PFAHNL (2014). 3D printing of surgical instruments for long-duration space missions, Aviat. Space Environ. Med. 85(7) (2014) 758-763.

[6] PERNICA, J., SUSTR, M., DOSTAL, P., BRABEC, M., DOBROCKY, D. (2021). Tensile Testing of 3D Printed Materials Made by Different Temperature. Manufacturing Technology, 2021, 21(3):398-404. DOI: $10.21062 / \mathrm{mft} .2021 .039$.

[7] M.K. THOMPSON, G. MORONI, T. VANEKER, G. FADEL, R.I. CAMPBELL, I. GIBSON, A. BERNARD, J. SCHULZ, P. GRAF, B. AHUJA (2016). Design for Additive Manufacturing: Trends, opportunities, considerations, and constraints, CIRP annals 65(2) (2016) 737-760.

[8] G.A.O. ADAM, D. ZIMMER (2015). On design for additive manufacturing: evaluating geometrical limitations, Rapid Prototyp. J. 21(6) (2015) 662-670.

[9] E. FODRAN, M. KOCH, U. MENON (1996). Mechanical and dimensional characteristics of fused deposition modeling build styles, $1996 \mathrm{In}$ ternational Solid Freeform Fabrication Symposium, 1996.

[10] D. ESPALIN, J. ALBERTO RAMIREZ, F. MEDINA, R. WICKER (2014). Multi-material, multi-technology FDM: exploring build process variations, Rapid Prototyp. J. 20(3) (2014) 236-244.

[11] F. ROGER, P. KRAWCZAK (2015). 3D-printing of thermoplastic structures by FDM using heterogeneous infill and multi-materials: An integrated design-advanced manufacturing approach for factories of the future, Congrès français de mécanique, AFM, Association Française de Mécanique, 2015.

[12] M.H. ALI, N. MIR-NASIRI, W.L. KO (2016). Multi-nozzle extrusion system for 3D printer and its control mechanism, Int. J. Adv. Manuf. Technol. 86(1) (2016) 999-1010.

[13] D. BACA, R. AHMAD (2020). The impact on the mechanical properties of multi-material polymers fabricated with a single mixing nozzle and multi-nozzle systems via fused deposition modeling, Int. J. Adv. Manuf. Technol. 106(9) (2020) 4509-4520.
[14] S. KHALIL, J. NAM, W. SUN (2005). Multinozzle deposition for construction of 3D biopolymer tissue scaffolds, Rapid Prototyp. J. 11(1) (2005) 9-17.

[15] Y. YAN, Z. XIONG, Y. HU, S. WANG, R. ZHANG, C. ZHANG (2003). Layered manufacturing of tissue engineering scaffolds via multi-nozzle deposition, Mater. Lett. 57(18) (2003) 2623-2628.

[16] E. MALONE, H. LIPSON (2008). Multi-material freeform fabrication of active systems, Engineering Systems Design and Analysis, 2008, pp. 345-353.

[17] M.A. JAFARI, W. HAN, F. MOHAMMADI, A. SAFARI, S.C. DANFORTH, N. LANGRANA (2000). A novel system for fused deposition of advanced multiple ceramics, $R a$ pid Prototyp. J. 6(3) (2000) 161-175.

[18] K. ARCAUTE, L. OCHOA, B.K. MANN, R.B. WICKER (2005) Stereolithography of PEG Hydrogel Multi-Lumen Nerve Regeneration Conduits, ASME 2005 International Mechanical Engineering Congress and Exposition, 2005, pp. 161-167.

[19] K. ARCAUTE, B.K. MANN, R.B. WICKER (2006). Stereolithography of three-dimensional bioactive poly (ethylene glycol) constructs with encapsulated cells, Ann. Biomed. Eng. 34(9) (2006) 1429-1441.

[20] T.J. COOGAN, D.O. KAZMER (2017). Healing simulation for bond strength prediction of FDM, Rapid Prototyp. J. (2017).

[21] P.K. GURRALA, S.P. REGALLA (2014). Part strength evolution with bonding between filaments in fused deposition modelling: This paper studies how coalescence of filaments contributes to the strength of final FDM part, Virtual. Phys. Prototyp. 9(3) (2014) 141-149.

[22] A. ABILGAZIYEV, T. KULZHAN, N. RAISSOV, M.H. ALI, W.L.K. MATCH, N. MIR-NASIRI (2015). Design and development of multi-nozzle extrusion system for 3D printer, 2015 International Conference on Informatics, Electronics \& Vision (ICIEV), 2015, pp. 1-5.

[23] K. VAN DE VELDE, P. KIEKENS (2002). Biopolymers: overview of several properties and consequences on their applications, Polym. Test. 21(4) (2002) 433-442. 